\title{
Cavity-loss-induced generation of entangled atoms
}

\author{
M. B. Plenio, S. F. Huelga,* A. Beige, and P. L. Knight \\ Optics Section, Blackett Laboratory, Imperial College London, London SW7 2BZ, England
}

(Received 14 September 1998)

\begin{abstract}
We discuss the generation of entangled states of two two-level atoms inside an optical resonator. When the cavity decay is continuously monitored, the absence of photon counts is associated with the presence of an atomic entangled state. In addition to being conceptually simple, this scheme can be demonstrated with presently available technology. We describe how such a state is generated through conditional dynamics, using quantum jump methods, including both cavity damping and spontaneous emission decay, and evaluate the fidelity and relative entropy of entanglement of the generated state compared with the target entangled state. [S1050-2947(99)08303-1]

PACS number(s): 42.50.Lc, 42.50.Dv, 03.67.-a
\end{abstract}

\section{INTRODUCTION}

Superposition effects in composite systems are well known in classical physics. However, when the superposition principle is combined with a tensor product structure for the space of states, an entirely quantum-mechanical effect arises: Quantum states can be entangled [1]. This fact was early recognized as the characteristic of the quantum formalism [2]. However, early work concentrated on the implications of entanglement on the nonlocal structure of quantum theory [3], and it was considered by many as a purely philosophical issue. The reason for the renewed interest in the fundamental aspects of quantum mechanics is twofold. On the one hand, it was discovered that Bell's inequalities do not provide a good criterion for discriminating between classical and quantum correlations when dealing with mixed states [4]. New criteria for characterizing the separability of a given quantum state have been proposed [5], and measures of entanglement have been introduced [6,7]. On the other hand, it has been realized that entangled states allow new practical applications, ranging from quantum computation [8] and secure cryptographic schemes [9] to improved optical frequency standards [10]. The feasibility of some these applications has been demonstrated in recent experiments [11]. In particular, recent advances in ion trapping technology [12] and cavity QED [13] provide suitable scenarios for manipulating small quantum systems.

In this paper we will discuss a scheme that allows the generation of a maximally entangled state of two two-level atoms within a single-mode cavity field. The underlying idea is conceptually simple, and relies on the concept of conditional dynamics due to continuous observation of the cavity field. The key to understanding how the entangled state is generated in this scheme is population trapping [14]. There are three dressed states of the combined two-atom plus cavity field mode system; one has a zero eigenvalue, which is therefore stationary, whereas the other two decay in time. Provided no photon leaks out of the cavity (which is why

\footnotetext{
*Permanent address: Departamento de Física, Avda de Calvo Sotelo n/s, 33007 Oviedo, Spain.
}

conditional dynamics is necessary), a pure entangled state between the two atoms results. From the experimental point of view, this proposal is feasible with presently available technology.

The paper is organized as follows. In Sec. II we describe the system of interest. This consists of two trapped atoms inside an optical resonator. Certain aspects of the dynamics of this system, when driven by an external field, have been addressed, for instance, in the context of the two-atom microlaser [15]. The coherence properties of the fluorescence from close-lying atoms in an optical cavity have been considered recently using the quantum jump approach [16]. Our proposal provides a probabilistic scheme [17] for generating an entangled state of the two atoms. This will require an initial preparation, which involves the selective excitation of one of the atoms and the continuous monitoring of photons leaking out of the cavity. The time evolution under the condition of no-photon detection is discussed in Sec. III. We will show that the quantum jump approach provides a suitable theoretical framework for analyzing the dynamics in a simple and intuitive way. The fidelity with respect to a maximally entangled state and the relative entropy of entanglement of the final atomic state will be evaluated in Sec. IV.

\section{DESCRIPTION OF THE PHYSICAL SYSTEM}

Our system consists of two two-level ions confined in a linear trap which has been surrounded by a leaky optical cavity. We will refer to atom $a$ and atom $b$ when the context requires us to differentiate them, but otherwise they are supposed to be identical. We denote the atomic ground and excited states by $|0\rangle_{i}$ and $|1\rangle_{i}$, and call $2 \Gamma\left(\Gamma=\Gamma_{a}=\Gamma_{b}\right)$ the spontaneous emission rate from the upper level. We assume that the distance between the atoms is much larger than an optical wavelength, and that therefore dipole-dipole interactions can be neglected [18]. In addition, this requirement allows us to assume that each atom can be individually addressed with laser light. The cavity mode is assumed to be resonant with the atomic transition frequency, and we will denote the cavity decay rate by $\kappa$. For the sake of generality we allow the coupling between each atom and the cavity 


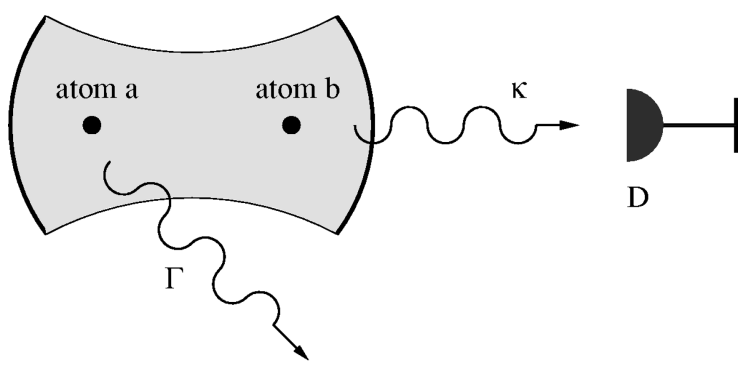

FIG. 1. Experimental setup. The system consists of two twolevel atoms placed inside a leaky cavity. The decay rate $\Gamma$ describes the spontaneous emission of the atoms, while the rate $\kappa$ refers to photons leaking through the cavity mirrors. The latter can be monitored by the detector $D$.

mode, $g_{i}$, to be different. ${ }^{1}$ The relaxation of the ion-cavity system can take place through two different channels, at rates $\kappa$ (cavity decay) and $\Gamma$ (spontaneous decay).

In what follows we will assume that the coupling constants and the decay rates are such that

$$
g_{i}, \kappa \gg \Gamma .
$$

The experimental setup is depicted in Fig. 1. Note the presence of a single photon detector $D$ in our scheme. This setup will allow us to monitor the decay of the system through the fast channel, i.e., photons leaking through the cavity mirrors. On the other hand, spontaneously emitted photons from the slow decay channel in the regime of Eq. (1), will not be detected. The initial state of the system is of the form

$$
|0\rangle \otimes|0\rangle_{a} \otimes|0\rangle_{b} \equiv|000\rangle,
$$

where the first index refers to the cavity field state. Now applying a $\pi$ pulse to atom $a$, we introduce an excitation into the system, and the initial conditions for our scheme will be given by the composite state

$$
\left|\psi_{0}\right\rangle=|0\rangle \otimes|1\rangle_{a} \otimes|0\rangle_{b} \equiv|010\rangle .
$$

In the following we will use Eq. (3) as the basis for all the following discussions. It is important to emphasize that our scheme only requires the atoms to be cooled to the LambDicke limit, i.e. each atom is localized within one wavelength of the emitted light. But no further cooling to the motional ground state is necessary. This notably simplifies the experimental realizability of the proposal.

Experiments on ions in optical cavities are underway, for example, in Innsbruck. In these experiments the $S_{1 / 2}-D_{5 / 2}$ transition of calcium ions [lifetime $(2 \Gamma)^{-1}=1 \mathrm{~s}$ ] couples to an optical cavity which has a decay rate $\kappa$ between 1 and 10 $\mathrm{kHz}$. The ions are separated by many optical wavelengths, and can therefore be addressed separately using focused laser beams [19].

\footnotetext{
${ }^{1} \mathrm{~A}$ symmetric location of the atoms with respect to the center of the trap suffices to make $g_{a}=g_{b}$. However, experimentally this may well be hard to achieve.
}

\section{ATOM-CAVITY SYSTEM WITHOUT DECAY}

In order to illustrate the main idea underlying this proposal, let us ignore any relaxation process for the moment. The unitary time evolution of the system will then be governed by the Hamiltonian

$$
\begin{aligned}
H= & \sum_{i=a, b} \hbar \omega_{i}|1\rangle_{i i}\langle 1|+\hbar \nu b^{\dagger} b+i \hbar \\
& \times \sum_{i=a, b}\left(g_{i} b|1\rangle_{i i}\langle 0|-\text { H.c. }\right),
\end{aligned}
$$

where $b$ and $b^{\dagger}$ denote the annihilation and creation operators for the single-mode cavity field. The fourth term in this expression is the familiar Jaynes-Cummings (JC) interaction between each atomic system and the cavity mode. Moving to an interaction picture with respect to the unperturbed Hamiltonian,

$$
H_{0}=\sum_{i=a, b} \hbar \omega_{i}|1\rangle_{i i}\langle 1|+\hbar \nu b^{\dagger} b,
$$

and assuming exact resonance between the cavity mode and the atomic transition, $\nu=\omega_{i}$, we find

$$
H_{I}=i \hbar \sum_{i=a, b}\left(g_{i} b|1\rangle_{i i}\langle 0|-\text { H.c. }\right)
$$

where the coupling constants $g_{i}$ have been taken to be real. In the basis $\mathcal{B}=(|100\rangle,|010\rangle,|001\rangle)$, the interaction picture Hamiltonian reads

$$
H_{I}=\frac{\hbar}{i}\left(\begin{array}{ccc}
0 & g_{a} & g_{b} \\
-g_{a} & 0 & 0 \\
-g_{b} & 0 & 0
\end{array}\right) .
$$

It is easy to check that the eigenvalues associated with this operator are given by

$$
\begin{gathered}
\lambda_{0}=0, \\
\lambda_{1,2}= \pm \hbar \sqrt{g_{a}^{2}+g_{b}^{2}},
\end{gathered}
$$

with corresponding eigenvectors

$$
\begin{gathered}
\left|\lambda_{0}\right\rangle=\frac{1}{\sqrt{g_{a}^{2}+g_{b}^{2}}}\left(g_{a}|001\rangle-g_{b}|010\rangle\right), \\
\left|\lambda_{1,2}\right\rangle=\frac{1}{\sqrt{2}}\left(|100\rangle \pm \frac{i}{\sqrt{g_{a}^{2}+g_{b}^{2}}}\left(g_{b}|001\rangle+g_{a}|010\rangle\right) .\right.
\end{gathered}
$$

Note that when $g_{a}=g_{b}$, the solution $\left|\lambda_{0}\right\rangle$ is a tensor product of the cavity field in the vacuum state and the maximally entangled atomic state

$$
\left|\phi^{-}\right\rangle=\frac{1}{\sqrt{2}}(|01\rangle-|10\rangle) .
$$

To prepare an entangled state of the atoms one now needs a mechanism that destroys the population of the cavity mode. 
One possibility is to use a leaking cavity, and to detect all photons coming through the cavity mirrors. If a photon is detected, the system is in the ground state $|000\rangle$. Then the experiment has to be repeated. But if not, the system goes over into a state which cannot decay. Therefore, the atoms should end up in state $\left|\lambda_{0}\right\rangle$, the entangled state, where the cavity mode is not populated.

Using the quantum jump approach, we will see that the dynamics under the condition that no photon has been detected outside the cavity is governed by an effective Hamiltonian whose solutions keep track of the structure illustrated above. More precisely, for sufficiently large times the state of the system will be a tensor product of the cavity field in the vacuum state and an entangled state of the two atoms.

\section{ATOM-CAVITY SYSTEM INCLUDING DECAY}

Let us now consider the experimental situation depicted in Fig. 1, in which the decay of the cavity field is monitored by means of the detector $D$. For the moment we will assume that the detector has $100 \%$ efficiency, but later this constraint will be relaxed. The time evolution is now governed by the Hamiltonian

$$
\begin{aligned}
H= & \sum_{i=a, b} \hbar \omega_{i}|1\rangle_{i i}\langle 1|+\hbar \nu b^{\dagger} b+\sum_{\mathbf{k} \lambda} \hbar \omega_{\mathbf{k} \lambda} a_{\mathbf{k} \lambda}^{\dagger} a_{\mathbf{k} \lambda}+i \hbar \sum_{i=a, b}\left(g_{i} b|1\rangle_{i i}\langle 0|-\text { H.c. }\right) \\
& +i \hbar \sum_{i=a, b} \sum_{\mathbf{k} \lambda}\left(g_{\mathbf{k} \lambda} a_{\mathbf{k} \lambda}|1\rangle_{i i}\langle 0| e^{i\left(\omega_{i}-\omega_{\mathbf{k} \lambda}\right) t}-\text { H.c. }\right)+i \hbar \sum_{\mathbf{k} \lambda}\left(s_{\mathbf{k} \lambda} a_{\mathbf{k} \lambda} b^{\dagger} e^{i\left(\nu-\omega_{\mathbf{k} \lambda}\right) t}-\text { H.c. }\right),
\end{aligned}
$$

where $a_{\mathbf{k} \lambda}^{\dagger}$ and $a_{\mathbf{k} \lambda}$ denote the free radiation field creation and annihilation operators of a photon in the mode (k, $\lambda$ ). The two remaining terms including the coupling constants $g_{\mathbf{k} \lambda}$ and $s_{\mathbf{k} \lambda}$ describe, respectively, the coupling of the atoms and the cavity mode to the free radiation field. The initial state of the system, $\left|\psi_{0}\right\rangle$, is given by Eq. (3). At a time $t$, and provided that no photon leaking through the cavity mirrors has been detected, the state of the system can be described in terms of a density operator of the form

$$
\rho\left(t, \psi_{0}\right)=\left(P_{0}\left(t, \psi_{0}\right)\left|\hat{\psi}_{\mathrm{coh}}(t)\right\rangle\left\langle\hat{\psi}_{\mathrm{coh}}(t)\left|+P_{\mathrm{spon}}\left(t, \psi_{0}\right)\right| 000\right\rangle\langle 000|\right) / \operatorname{tr}() .
$$

Here $P_{0}\left(t, \psi_{0}\right)$ is the probability for no photon emission, where neither the cavity field nor the atoms have decayed until $t$, and $\left|\hat{\psi}_{\text {coh }}(t)\right\rangle$ denotes the normalized state resulting from the coherent evolution in this case. Later we will also use the notation $\left|\psi_{\text {coh }}\right\rangle$ for the unnormalized state. The second term of the mixture takes into account that spontaneously emitted photons are not observed. If an atom emits a spontaneous photon, then the state of the atom-cavity system is reduced to the state $|000\rangle$. Our main task consists of evaluating the explicit form of the state $\left|\hat{\psi}_{\mathrm{coh}}(t)\right\rangle$ of $P_{0}\left(t, \psi_{0}\right)$, and the probability $P_{\text {spon }}\left(t, \psi_{0}\right)$ for spontaneously decay in $(0, t)$. The quantum jump approach (also called the quantum trajectories method) [20-22] (See Ref. [23] for a recent review) provides a suitable theoretical framework for this analysis.

\section{A. Derivation of the conditional time evolution}

Let us consider an idealized situation where both the photons leaking through the cavity and the spontaneously emitted photons could be detected. In the derivation of the quantum jump approach, one envisages an equally spaced sequence of gedanken photon measurements at times $t_{1}, t_{2}, \ldots, t_{n-1}, t_{n}$, such that $t_{i}-t_{i-1}=\Delta t$. According to the projection postulate, the subensemble for which no photon has been detected until time $t_{n}$ is described by the (unnormalized) state vector

$$
\left|\psi_{\mathrm{coh}}(t)\right\rangle=\mathrm{P}_{0} U\left(t_{n}, t_{n-1}\right) \mathrm{P}_{0} \ldots \mathrm{P}_{0} U\left(t_{1}, t_{0}\right)\left|0_{\mathrm{ph}}\right\rangle\left|\psi\left(t_{0}\right)\right\rangle \equiv\left|0_{\mathrm{ph}}\right\rangle U_{\text {cond }}\left(t_{n}, t_{0}\right)\left|\psi\left(t_{0}\right)\right\rangle,
$$

where we have defined the projector

$$
\mathrm{P}_{0}=\left|0_{\mathrm{ph}}\right\rangle \mathbb{I}_{A}\left\langle 0_{\mathrm{ph}}\right|
$$

and $\mathbb{I}_{A}$ denotes the identity over the atomic variables. Therefore, the operator $U_{\text {cond }}\left(t_{n}, t_{0}\right)$ describes the time evolution of the system under the condition that no photon has been detected. Using our previous notation, the state of the system at a time $t_{n}$ will be given by $U_{\text {cond }}\left(t_{n}, t_{0}\right)\left|\psi\left(t_{0}\right)\right\rangle$ when the system has not relaxed through either the fast or the slow channel. Taking into account Eq. (12) and the form of the projector $\mathbb{P}_{0}$, our problem reduces to evaluating expressions of the form $\left\langle 0_{\mathrm{ph}}\left|U\left(t_{n}, t_{n-1}\right)\right| 0_{\mathrm{ph}}\right\rangle$, which can be done easily using second-order perturbation theory. The calculations can be simplified moving to an appropriate interaction picture with respect to the unperturbed Hamiltonian

$$
H_{0}=\sum_{i=a, b} \hbar \omega_{i}|1\rangle_{i i}\langle 1|+\hbar \nu b^{\dagger} b+\sum_{\mathbf{k} \lambda} \hbar \omega_{\mathbf{k} \lambda} a_{\mathbf{k} \lambda}^{\dagger} a_{\mathbf{k} \lambda}
$$

In second-order perturbation theory one obtains 


$$
\left\langle 0_{\mathrm{ph}}\left|U\left(t_{n}, t_{n-1}\right)\right| 0_{\mathrm{ph}}\right\rangle=1-\frac{1}{\hbar} \int_{t_{n-1}}^{t_{n}} d t^{\prime}\left\langle 0_{\mathrm{ph}}\left|H_{I}\left(t^{\prime}\right)\right| 0_{\mathrm{ph}}\right\rangle-\frac{1}{\hbar^{2}} \int_{t_{n-1}}^{t_{n}} d t^{\prime} \int_{t_{n-1}}^{t^{\prime}} d t^{\prime \prime}\left\langle 0_{\mathrm{ph}}\left|H_{I}\left(t^{\prime}\right) H_{I}\left(t^{\prime \prime}\right)\right| 0_{\mathrm{ph}}\right\rangle
$$

where the interaction Hamiltonian reads

$$
\begin{aligned}
H_{I}=H_{a-c}+H_{a-f}+H_{c-f}= & i \hbar \sum_{i=a, b}\left(g_{i} b|1\rangle_{i i}\langle 0|-\text { H.c. }\right)+i \hbar \sum_{i=a, b} \sum_{\mathbf{k} \lambda}\left(g_{\mathbf{k} \lambda} a_{\mathbf{k} \lambda}|1\rangle_{i i}\langle 0| e^{i\left(\omega_{i}-\omega_{\mathbf{k} \lambda}\right) t}-\text { H.c. }\right) \\
& +i \hbar \sum_{\mathbf{k}, \lambda}\left(s_{\mathbf{k} \lambda} a_{\mathbf{k} \lambda} b^{\dagger} e^{i\left(\nu-\omega_{\mathbf{k} \lambda}\right) t}-\text { H.c. }\right)
\end{aligned}
$$

In first-order perturbation theory, only the JC term contributes to Eq. (17) since both $\left\langle 0_{\mathrm{ph}}\left|a_{\mathbf{k} \lambda}\right| 0_{\mathrm{ph}}\right\rangle$ and $\left\langle 0_{\mathrm{ph}}\left|a_{\mathbf{k} \lambda}^{\dagger}\right| 0_{\mathrm{ph}}\right\rangle$ are zero. On the other hand, the second-order contribution from the JC term is quadratic in $g \Delta t$ and can be neglected. A contribution from the term $H_{a-f}^{i}(i=a, b)$ appears only in second-order perturbation theory and can be evaluated using the usual Markov approximation [24]. Then one finds

$$
\begin{aligned}
& -\frac{1}{\hbar^{2}} \int_{t_{n-1}}^{t_{n}} d t^{\prime} \int_{t_{n-1}}^{t^{\prime}} d t^{\prime \prime}\left\langle 0_{\mathrm{ph}}\left|H_{a-f}\left(t^{\prime}\right) H_{a-f}\left(t^{\prime \prime}\right)\right| 0_{\mathrm{ph}}\right\rangle \\
& \quad=-\Gamma_{i}|1\rangle_{i i}\langle 1| \Delta t
\end{aligned}
$$

where

$$
\Gamma_{i}=\frac{e^{2}}{6 \pi \epsilon_{0} \hbar c^{3}} d^{2} \omega_{i}^{3}
$$

Similarly, one can show that the term $H_{c-f}$ yields a formally analogous contribution, now replacing the atomic decay rate by the cavity decay rate $\kappa$. The form of the conditional Hamiltonian is now easily inferred, taking into account that

$$
\begin{aligned}
& \prod_{i=1}^{n}\left\langle 0_{\mathrm{ph}}\right| U\left(t_{n}, t_{n-1}\left|0_{\mathrm{ph}}\right\rangle\right. \\
& \quad=U_{\text {cond }}\left(t_{n}, 0\right)=\mathcal{T} \exp \left(-\frac{i}{\hbar} \int_{0}^{t_{n}} d t^{\prime} H_{\text {cond }}\left(t^{\prime}\right)\right),
\end{aligned}
$$

where $\mathcal{T}$ indicates a time ordered expression. We find

$$
H_{\text {cond }}=\frac{\hbar}{i}\left(\begin{array}{ccc}
\kappa & g_{a} & g_{b} \\
-g_{a} & \Gamma & 0 \\
-g_{b} & 0 & \Gamma
\end{array}\right) \equiv \frac{\hbar}{i} M
$$

in the basis $\mathcal{B}=(|100\rangle,|010\rangle,|001\rangle)$. The corresponding eigenvalues of $M$ are given by

$$
\lambda_{0}=\Gamma
$$

$$
\lambda_{1,2}=(\kappa+\Gamma \pm i S) / 2,
$$

with $S=\sqrt{4\left(g_{a}^{2}+g_{b}^{2}\right)-(\kappa-\Gamma)^{2}}$. The eigenvector of the smallest eigenvalue is the same entangled state as in Eq. (10), i.e.,

$$
\left|\lambda_{0}\right\rangle=\frac{1}{\sqrt{g_{a}^{2}+g_{b}^{2}}}\left(g_{a}|001\rangle-g_{b}|010\rangle\right)
$$

$M$ has three normalized eigenvectors $\left|\lambda_{i}\right\rangle$, which are in general not orthogonal. The reciprocal vectors $\left\langle\lambda^{i}\right|$ are defined by $\left\langle\lambda^{i} \mid \lambda_{j}\right\rangle=\delta_{i j}$. Then one can write $M=\Sigma_{i} \lambda_{i}\left|\lambda_{i}\right\rangle\left\langle\lambda^{i}\right|$. For the conditional time evolution operator, one has the representation

$$
U_{\text {cond }}(t, 0)=e^{-M t}=\sum_{i=1}^{3} e^{-\lambda_{i} t}\left|\lambda_{i}\right\rangle\left\langle\lambda^{i}\right|
$$

Therefore, provided that no photon has been detected during the time interval $[0, t]$ and $t$ satisfies

$$
\Gamma^{-1} \gg t \gg \kappa^{-1}
$$

the exponentials $\exp \left(-\lambda_{1 / 2} t\right)$ can be neglected while $\exp \left(-\lambda_{0} t\right)$ is still close to unity and the system will be in the state

$$
\left|\hat{\psi}_{\text {coh }}(t)\right\rangle=U_{\text {cond }}(t, 0)\left|\psi_{0}\right\rangle=e^{-\lambda_{0} t}\left|\lambda_{0}\right\rangle\left\langle\lambda^{0} \mid \psi_{0}\right\rangle /\|\|=\left|\lambda_{0}\right\rangle .
$$

This state factorizes as a tensor product between the cavity field in the vacuum state and an entangled state of the two atoms.

More precisely, the conditional time evolution operator $U_{\text {cond }}$ can be calculated as

$$
e^{-M t}=\frac{\left(M-\lambda_{1}\right)\left(M-\lambda_{2}\right)}{\left(\lambda_{0}-\lambda_{1}\right)\left(\lambda_{0}-\lambda_{2}\right)} e^{-\lambda_{0} t}+(\text { cyclic permutations }),
$$

which can easily be verified by application to the eigenvectors [25]. Applying this operator to our initial state, Eq. (3), we obtain 


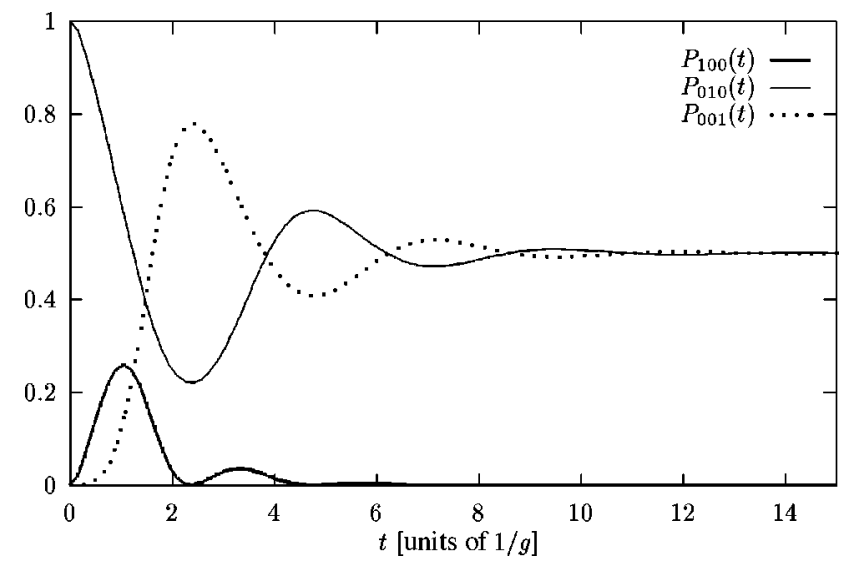

FIG. 2. The time dependence of the probability amplitudes for the basis states $|100\rangle,|010\rangle$, and $|001\rangle$ under the conditional time evolution that no photon has been detected at all. We have chosen $g_{a}=g_{b}=g=\kappa$ and $\Gamma=10^{-3} g$. After a short time the cavity mode is decayed, and the atoms have reached the pure entangled atomic state.

$$
\begin{aligned}
\left|\hat{\psi}_{\mathrm{coh}}(t)\right\rangle= & \frac{1}{g_{a}^{2}+g_{b}^{2}}\left[g_{b} e^{-\Gamma t}\left(\begin{array}{r}
0 \\
g_{b} \\
-g_{a}
\end{array}\right)+g_{a} e^{-(1 / 2)(\kappa+\Gamma) t}\right. \\
& \times\left\{\left(\begin{array}{c}
0 \\
g_{a} \\
g_{b}
\end{array}\right) \cos (S t / 2)+\frac{1}{S}\left(\begin{array}{r}
-2\left(g_{a}^{2}+g_{b}^{2}\right) \\
g_{a}(\kappa-\Gamma) \\
g_{b}(\kappa-\Gamma)
\end{array}\right)\right. \\
& \times \sin (S t / 2)\} .
\end{aligned}
$$

The probability amplitudes for the three basis states are plotted in Fig. 2. As expected, on a time scale such that $\Gamma^{-1} \gg t \gg \kappa^{-1}$, the contribution from terms multiplied by a damping factor proportional to the sum $\kappa+\Gamma$ becomes negligible, and the conditional state vector is a two-particle entangled state correlated with the cavity field in the vacuum state $\left|\lambda_{0}\right\rangle$.

\section{B. Calculation of the detection probabilities}

After the derivation of the conditional time evolution, we are now in a position to calculate the probabilities for photon emissions. We first calculate the probability that there is no decay at all, neither spontaneous emissions by the atoms nor photons leaking out of the cavity. Subsequently we will derive the probability for (a) having a spontaneous decay from the atoms, and (b) for having photon emission from the cavity.

The probability to have no photon emission (neither spontaneously emitted nor leaking through the cavity mirrors) until time $t$ is given by the norm squared of Eq. (30), i.e.

$$
P_{0}\left(t, \psi_{0}\right)=\| U_{\text {cond }}(t, 0)\left|\psi_{0}\right\rangle \|^{2} \text {. }
$$

This general expression can be simplified considerably for large times $t$. The probability to detect no photon until time $t$ with $t \gg \kappa^{-1}$ is equal to

$$
P_{0}\left(t, \psi_{0}\right)=\frac{g_{b}^{2}}{g_{a}^{2}+g_{b}^{2}} e^{-2 \Gamma t}
$$

In our experimental setup (see Fig. 1), only photons leaking through the cavity mirrors are monitored and, as we have pointed out, the state of the system will be the mixture given by Eq. (13). The quantum jump approach [21-23] provides a transparent way to evaluate the weight of the component $|000\rangle$, i.e., the probability for a spontaneous emission from an atom.

Let us denote by $t^{\prime}$ an intermediate time within the interval $[0, t]$. The probability $P$ of having an emission at any time in that interval will be given by

$$
P=\int_{0}^{t} d t^{\prime} w_{1}\left(t^{\prime}, \psi_{0}\right)
$$

where $w_{1}\left(t^{\prime}, \psi_{0}\right)$ denotes the probability density for the first photon at time $t^{\prime}$ for the given initial state $\left|\psi_{0}\right\rangle[26,27]$. Since $w_{1}\left(t^{\prime}, \psi_{0}\right) d t$ equals $P_{0}\left(t^{\prime}, \psi_{0}\right)-P_{0}\left(t^{\prime}+d t^{\prime}, \psi_{0}\right)$, one has

$$
\begin{aligned}
w_{1}\left(t^{\prime}, \psi_{0}\right) & =-\frac{d}{d t^{\prime}} P_{0}\left(t^{\prime}, \psi_{0}\right) \\
& =\left\langle\psi_{0}\left|e^{-M^{\dagger} t^{\prime}}\left(M+M^{\dagger}\right) e^{-M t^{\prime}}\right| \psi_{0}\right\rangle .
\end{aligned}
$$

Taking into account the explicit form of $M$ in Eq. (22), we find

$$
w_{1}\left(t^{\prime}, \psi_{0}\right)=2 \kappa\left|\left\langle 100\left|U_{\text {cond }}\left(t^{\prime}, 0\right)\right| \psi_{0}\right\rangle\right|^{2}+2 \Gamma\left(\left|\left\langle 010\left|U_{\text {cond }}\left(t^{\prime}, 0\right)\right| \psi_{0}\right\rangle\right|^{2}+\left|\left\langle 001\left|U_{\text {cond }}\left(t^{\prime}, 0\right)\right| \psi_{0}\right\rangle\right|^{2}\right) \text {. }
$$

As expected, both relaxation channels contribute separately to the decay rate $w_{1}$. Setting $t^{\prime}$ equal to 0 , one finds that the probability density for a photon leaking through the cavity mirrors is given by the population of the state $|100\rangle$ multiplied by the cavity decay rate. Similarly, the probability for spontaneous emission is determined by the population of the states $|010\rangle$ and $|001\rangle$.

In our case we are only interested in the contribution to $P$ in Eq. (33) coming from spontaneously emitted photons. Using Eq. (33), one finds

$$
P_{\text {spon }}\left(t, \psi_{0}\right)=2 \Gamma \int_{0}^{t} d t^{\prime}\left(\left|\left\langle 010\left|U_{\text {cond }}\left(t^{\prime}, 0\right)\right| \psi_{0}\right\rangle\right|^{2}+\left|\left\langle 001\left|U_{\text {cond }}\left(t^{\prime}, 0\right)\right| \psi_{0}\right\rangle\right|^{2}\right) \text {. }
$$


However, from the point of view of simplifying the calculations, it is easier to evaluate the probability of cavity decay. In a similar way one obtains

$$
P_{\text {cav }}\left(t, \psi_{0}\right)=2 \kappa \int_{0}^{t} d t^{\prime}\left|\left\langle 010\left|U_{\text {cond }}\left(t^{\prime}, 0\right)\right| \psi_{0}\right\rangle\right|^{2}
$$

Taking into account the results of the previous section for the unnormalized state $\left|\psi_{\text {coh }}\right\rangle$, we can write

$$
P_{\text {cav }}\left(t, \psi_{0}\right)=\frac{\kappa g_{a}^{2}}{(\kappa+\Gamma)\left(g_{a}^{2}+g_{b}^{2}+\kappa \Gamma\right)}\left[1-\frac{e^{-(\kappa+\Gamma) t}}{S^{2}}\left[4\left(g_{a}^{2}+g_{b}^{2}+\kappa \Gamma\right)+(\kappa+\Gamma)(S \sin (S t)-(\kappa+\Gamma) \cos (S t))\right]\right]
$$

and calculate $P_{\text {spon }}$ as the difference between unity and the sum $P_{0}+P_{\text {cav }}$.

\section{FIDELITY AND ENTANGLEMENT IN THE ASYMPTOTIC REGIME}

In Sec. II, we have derived exact analytical expressions for the no-decay probabilities. In this section we will now discuss these exact expressions in the asymptotic regime, i.e., for times longer than the cavity lifetime. Finally, we will characterize the quality of the entanglement generation by cavity loss in two ways. We will calculate the fidelity with respect to the maximally entangled state $\left|\phi^{-}\right\rangle$, and explicitly calculate a measure of entanglement (the relative entropy of entanglement [7]) for the state of the system.

In Fig. 3 we plot the probability $P_{\text {cav }}\left(t, \psi_{0}\right)$ that a photon has leaked out of the cavity. As expected, this function saturates at a point close to 0.5 when $g_{a}=g_{b}$ and $\Gamma$ is small. The reason for this is that the overlap of the initial state $|010\rangle$ with the singlet state $|0\rangle\left|\phi^{-}\right\rangle$is precisely $\frac{1}{2}$. If a photon leaks the cavity, then the atomic state is $|00\rangle$, i.e., the atomic state is a product state. If no photon leaks out of the cavity then the atoms are in an entangled state. Therefore, the scheme presented here succeeds in $50 \%$ of the cases. In the asymptotic regime we can write

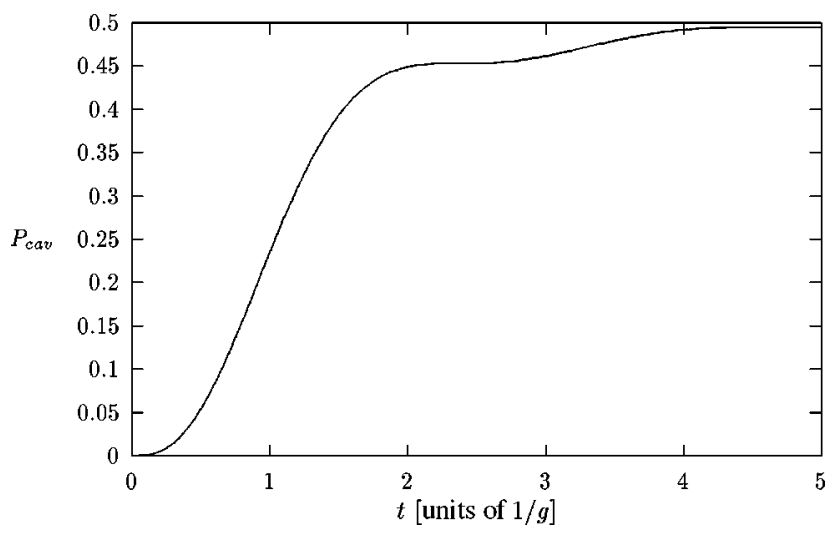

FIG. 3. The probability for the photon leaking through the cavity mirrors in the time interval $[0, t]$. We have chosen $g_{a}=g_{b}=g$ $=\kappa$ and $\Gamma=10^{-3} \mathrm{~g}$. For these parameters the cavity mode decays with a probability close to $\frac{1}{2}$. After a short time the state inside the cavity is stable.

$$
P_{\mathrm{spon}}\left(t, \psi_{0}\right)=1-\frac{g_{b}^{2}}{g_{a}^{2}+g_{b}^{2}} e^{-2 \Gamma t}-\frac{g_{a}^{2} \kappa}{(\Gamma+\kappa)\left(g_{a}^{2}+g_{b}^{2}+\Gamma \kappa\right)}
$$

Using the expressions for $\left|\psi_{\text {coh }}(t)\right\rangle$ and $P_{\text {spon }}\left(t, \psi_{0}\right)$ we can now calculate the state of the atoms at time $t$. This expression can then be used to evaluate the fidelity with respect to the maximally entangled state $\left|\phi^{-}\right\rangle$of Eq. (11). This result is represented in Fig. 4. We observe that for short times $t$ satisfying Eq. (27), the fidelity is almost unity. For times comparable to or larger than $\Gamma^{-1}$, the fidelity falls off exponentially. For our proposal only the region with small $t$ is relevant, so that the exponential decay of the fidelity for larger $t$ does not limit the efficiency of our scheme. In Fig. 4 we also plot the fidelity for imperfect counter efficiency (in this figure it is $\eta=0.8$ ). We observe that the fidelity is still high.

When dealing with entangled states it is interesting to know the amount of entanglement that is contained in a state. Especially for mixed states this is not directly related to the fidelity of the state. However, there exist quantitative entanglement measures for mixed states. In the following we will calculate the relative entropy of entanglement for the

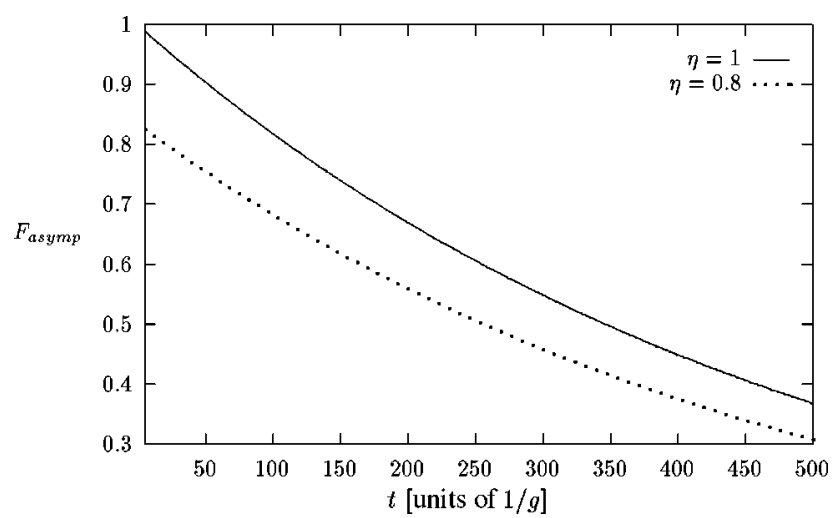

FIG. 4. Fidelity of the final atomic state with respect to the singlet state in the asymptotic limit, where $t$ is large compared with $\kappa^{-1}$. The dotted line corresponds to the case of a detector with finite efficiency $\eta$ (here $\eta=0.8$ ). For small times the fidelity of the atomic state with respect to the singlet state is high, even for a counter efficiency of $\eta=0.8$ ). For larger times the fidelity decreases exponentially because of a spontaneously emitted photon. 


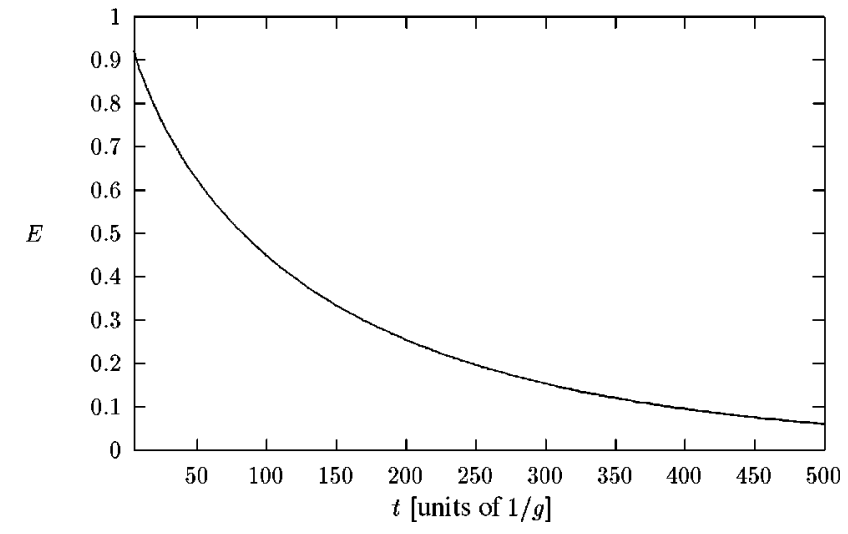

FIG. 5. Relative entropy of entanglement for the final mixed state in the asymptotic limit, where $t$ is large compared with $\kappa^{-1}$. As before, we have taken $g_{a}=g_{b}=g=\kappa$ and $\Gamma=10^{-3} g$. As long as the entangled state of the atoms does not decay spontaneously, the entropy $E$ is high.

states generated by our scheme. Due to the special form of the density operator $\rho$ of the two atoms,

$$
\begin{aligned}
\rho= & \frac{1}{P_{0}\left(t, \psi_{0}\right)+P_{\text {spon }}\left(t, \psi_{0}\right)}\left(P_{0}\left(t, \psi_{0}\right)\left|\phi^{-}\right\rangle\left\langle\phi^{-}\right|\right. \\
& \left.+P_{\text {spon }}\left(t, \psi_{0}\right)|00\rangle\langle 00|\right)
\end{aligned}
$$

it is possible to compute the relative entropy of entanglement of the final state [7] analytically. It is given by

$$
E(\rho)=(\lambda-2) \ln _{2}(1-\lambda / 2)+(1-\lambda) \ln _{2}(1-\lambda),
$$

where $\lambda=P_{0} /\left(P_{0}+P_{\text {spon }}\right)$. We have plotted this result in Fig. 5 for perfect counter efficiency. For short times (which are nevertheless longer than the cavity lifetime) the amount of entanglement is high, while it falls off exponentially for larger times. It should be noted that Eq. (40) contains entanglement for arbitrary counter efficiencies and spontaneous decay rates of the atoms. Therefore, our scheme is not limited by these experimental imperfections.

The fidelity of the mixed state $\rho$ can be determined experimentally using the technique recently developed by the National Institute of Standards group in Colorado [28] who used the fact that an atom singlet state is invariant under the radiation of both atoms with an identical laser. Both the diagonal elements and the relevant off-diagonal coherences of mixed states of the form of Eq. (40) can be measured by this method. Note that our approach allows us to incorporate easily a nonunit efficiency for the photodetectors. All we have to do is to modify the weight of the component $|000\rangle$ to account for the fact that there is a finite probability $\eta$ that the photodetector has not triggered in spite of the fact that leaking has occurred. The weight $P_{\text {spon }}$ is then replaced by $P_{\text {spon }}+(1-\eta) P_{\text {cav }}[26]$. The effect of nonideal detectors on the fidelity of the state is illustrated by the dotted line in Fig. 3 . For a counter efficiency of $80 \%$ the fidelity of the atomic state with respect to the singlet state is still high. Note that the effect of a nonperfect counter or spontaneous emission can be corrected using the following idea. A nonperfect counter or spontaneous emission lead to a $|000\rangle$ contribution in the density operator; see Eq. (40). If we irradiate a system in state $|000\rangle$ by a laser, cavity photons will be excited which will eventually leak out of the cavity mirror where they will be detected. The singlet contribution to the density operator remains invariant under the same procedure. In the state Eq. (40) only the $|000\rangle$ contribution will lead to the detection of a cavity photon. If we detect such a photon, the state of the system is projected to the state $|000\rangle$. If we fail to detect a photon, then, even for imperfect counters, we will end up in a state that has a higher proportion of the singlet state. Few repetitions of this procedure reduce the $|000\rangle$ contribution in the density operator of the atoms to very low values. Therefore, we conclude that our scheme is not overly sensitive to the counter efficiency.

\section{CONCLUSIONS}

We have described an experimental situation where entanglement between two atomic systems can be induced via continuous observation of the cavity loss. This proposal allows us to illustrate the effects of conditional time evolution and the power of the quantum jump approach as an analytical tool. From the experimental point of view the proposal has a number of advantages that should make its experimental realization possible with existing experimental methods [19].

(1) There exist open ion traps that allow us to implement a sufficiently small cavity. This will allow us to achieve high coupling constants between atoms and cavity.

(2) The conditions given by Eq. (1) are experimentally achievable, as we do not require a strong-coupling regime.

(3) The atoms only need to be cooled to the Lamb-Dicke limit [29]. In present ion trap implementations of entanglement manipulations, cooling to the motional ground state of the ions is required. For more than a single ion this can, at present, only be achieved with a finite precision, and currently represents a strong limit to the achievable fidelity of the state of the entangled atoms [28].

(4) The detection efficiency varies with the wavelength, but it can be up to $90 \%$. Although the amount of entanglement in the atomic state decreases with decreasing counter efficiency, it never vanishes (see also Fig. 4).

In addition, the initial preparation requires only a single laser pulse to excite selectively one of the atoms. Therefore, the experiment proposed here does seem feasible with presently available technology.

\section{ACKNOWLEDGMENTS}

We thank J. Steinbach and D. M. Segal for useful comments on the subject of this paper. Part of this work has been done during the 1998 Quantum Optics and Quantum Computing Workshop at the Benasque Center of Physics. This work was supported by the European Community, the U.K. Engineering and Physical Sciences Research Council, by a Feodor Lynen grant from the Alexander von Humboldt Foundation, and by the Leverhulme Trust. 
[1] M.B. Plenio and V. Vedral, Contemp. Phys. 39, 431 (1998).

[2] E. Schrödinger, Naturwissenschaften 23, 807 (1935) [an English translation appears in Quantum Theory and Measurement, edited by J. A. Wheeler and W.H. Zurek (Princeton University Press, Princeton, 1983), pp. 152-157]; A. Einstein, B. Podolsky, and N. Rosen, Phys. Rev. 47, 777 (1935).

[3] J. S. Bell, Rev. Mod. Phys. 38, 447 (1966).

[4] R. F. Werner, Phys. Rev. A 40, 4277 (1989); N. Gisin, Phys. Lett. A 210, 151 (1996).

[5] A. Peres, Phys. Rev. A 54, 2685 (1996); M. Horodecki, P. Horodecki, and R. Horodecki, Phys. Lett. A 223, 1 (1997).

[6] C. H. Bennett, D. P. DiVincenzo, J. Smolin, and W. K. Wootters, Phys. Rev. A 54, 3824 (1996); W. Wootters, Phys. Rev. Lett. 80, 2245 (1998); V. Vedral, M. B. Plenio, M. A. Rippin, and P. L. Knight, ibid. 78, 2275 (1997); V. Vedral, M. B. Plenio, K. Jacobs, and P. L. Knight, Phys. Rev. A 56, 4452 (1997); J. Eisert and M. B. Plenio, J. Mod. Opt. (to be published)

[7] V. Vedral and M. B. Plenio, Phys. Rev. A 57, 1619 (1998).

[8] A. Barenco, Contemp. Phys. 37, 375 (1996); V. Vedral and M. B. Plenio, Prog. Quantum Electron. 22, 1 (1998).

[9] C.H. Bennett and G. Brassard, in Proceedings of the IEEE International Conference on Computers, Systems and Signal Processing, Bangalore, India (IEEE, New York, 1984), p. 175; A. K. Ekert, Phys. Rev. Lett. 67, 661 (1991).

[10] J. J. Bollinger, W. M. Itano, D. J. Wineland, and D. J. Heinzen, Phys. Rev. A 54, R4649 (1996); S. F. Huelga, C. Macchiavello, T. Pellizzari, A. K. Ekert, M. B. Plenio, and J. I. Cirac, Phys. Rev. Lett. 79, 3865 (1997).

[11] C. Monroe, D. M. Meekhof, B. E. King, W. M. Itano, and D. J. Wineland, Phys. Rev. Lett. 75, 4714 (1995); Q. Turchette, C. J. Hood, W. Lange, H. Mabuchi, and H. J. Kimble, ibid. 75, 4710 (1995); X. Maitre et al., ibid. 79, 769 (1997); I. Chuang, L. M. K. Vandersypen, X. L. Zhou, D. W. Leung, and S. Lloyd, Nature (London) 393, 143 (1998); J. Jones, M. Mosca, and R. H. Hansen, ibid. 393, 344 (1998).

[12] D. W. Wineland, C. Monroe, W. M. Itano, D. Leibfried, B. E. King, and D. M. Meekhof, J. Res. Natl. Inst. Stand. Technol. 103, 259 (1998), and references therein.

[13] S. Haroche, M. Brune, and J. M. Raimond, Philos. Trans. R. Soc. London, Ser. A 355, 2367 (1997), and references therein.
[14] P. M. Radmore and P. L. Knight, J. Phys. B 15, 561 (1982).

[15] P. Horak and K. M. Gheri, Phys. Rev. A 53, R1970 (1996); K. M. Gheri, P. Horak, and H. Ritsch, J. Mod. Opt. 44, 605 (1997).

[16] P. Kochan, H. J. Carmichael, P. R. Morrow, and M. G. Raizen, Phys. Rev. Lett. 75, 45 (1995).

[17] Other probabilistic schemes for the generation of entangled states of massive particles can be found in E. S. Fry, T. Walther, and S. Li, Phys. Rev. A 52, 4381 (1995); E. Hagley, X. Maitre, G. Nogues, C. Wunderlich, M. Brune, J. M. Raimond, and S. Haroche, Phys. Rev. Lett. 79, 1 (1997).

[18] The influence of the dipole-dipole interaction when the atoms become closer will be analyzed elsewhere.

[19] R. Blatt (private communication).

[20] C. Cohen-Tannoudji and J. Dalibard, Europhys. Lett. 1, 441 (1986); J. Dalibard, Y. Castin, and K. Molmer, Phys. Rev. Lett. 68, 580 (1992).

[21] G. C. Hegerfeldt and T. S. Wilser, in Proceedings of the II International Wigner Symposium, Goslar 1991, edited by H. D. Doebner, W. Scherer, and F. Schroeck (World Scientific, Singapore, 1992).

[22] H. J. Carmichael, An Open Systems Approach to Quantum Optics, Lecture Notes in Physics, Vol. xx (Springer, Berlin, 1993).

[23] M. B. Plenio and P. L. Knight, Rev. Mod. Phys. 70, 101 (1998).

[24] C. Cohen-Tannoudji, J. Dupont-Roc, and G. Grynberg, AtomPhoton Interactions (Wiley, New York, 1992).

[25] For the general case see, e.g., F. R. Gantmacher, Matrizentheorie (Springer, Berlin, 1986).

[26] G. C. Hegerfeldt and M. B. Plenio, Phys. Rev. A 53, 1164 (1996).

[27] The quantity $w_{1}\left(t, \psi_{0}\right)$ has been discussed in more detail, for instance, by M. S. Kim, P. L. Knight, and K. Wodkiewicz, Opt. Commun. 62, 385 (1987); M. S. Kim and P. L. Knight, Phys. Rev. A 40, 215 (1989).

[28] Q. Turchette, C. S. Wood, B. E. King, C. J. Myatt, D. Liebfried, W. M. Itano, C. Monroe, and D. J. Wineland, Phys. Rev. Lett. 81, 3631 (1998).

[29] G. J. Yang, O. Zobay, and P. Meystre, Lanl e-print quant-ph/9811035. 